\title{
Anaesthesia management of a patient with large atrial septal defect with moderate pulmonary
}

\begin{abstract}
Atrial septal defect (ASD) is a common cardiac anomaly that may be first encountered in the adults and occurs more frequently in females. ASD accounts for $10 \%$ of congenital cardiac defects in adults. A 50 year old female patient posted for Total Abdominal Hysterectomy was diagnosed to have ostium secundum large ASD with mild tricuspid regurgitation with moderate pulmonary hypertension and mild essential hypertension. We report the successful management of the case using combined general anaesthesia with Bilateral Transversus Abdominis plane (TAP) block for postoperative analgesia. During the general anesthesia for the procedure our objectives were to avoid hypotension, hypoxemia, hypercarbia, hypothermia, reversal of shunt and fluid overload and postoperative analgesia by TAP block, The patient had an eventful perioperative course and discharged from the hospital on the $5^{\text {th }}$-postoperative day in good physical condition.
\end{abstract}

Volume 2 Issue 6 - 2015

\author{
Ritesh Roy, 'Anju Prasad ${ }^{2}$ \\ 'Department of Anaesthesia, Hi-Tech Medical College \& \\ Hospital, India \\ ${ }^{2}$ Department of Pharmacology, Hi-Tech Medical College \& \\ Hospital, India
}

Correspondence: RRitesh Roy, Plot No: 582, Jagannath Complex, Flat No: I02, Sahid Nagar, Bhubnaeswar-75I007, Odisha, India,Tel 09437101886,Email drriteshroy@yahoo.com

Received: August 02, 2015 | Published: August 21, 2015

Keywords: atrial septal defect, pulmonary arterial hypertension, abdominal hysterectomy, hypotension, hypoxemia, hypercarbia, tap block

Abbreviations: ASD, atrial septal defect; TAP, transversus abdominis plane; SVR, systemic vascular resistance; LOR, loss of resistance

\section{Introduction}

The most common acyanotic congential anomaly in adults is Atrial septal defect (ASD) with high prevalence in females. It accounts for $10 \%$ of acyanotic congential cardiac anomaly in adults. There are three different types of atrial septal defect. The Ostium primum type of defect occurs near the AV valve. The Sinus venosus type occur near the entry of superior venacava into the right atrium. The most common type is the Osteum secundum ASD which accounts for $70 \%$ of cases with a male: female ratio of $1: 2 .{ }^{1}$ It is midseptal in location and involves the fossa ovalis. Patients with ASD are usually asymptomatic in early life although there may be some physical underdevelopment and increased tendency for respiratory infection. ASD causes shunting of blood from left to right causing right ventricular volume overload and hypertrophy, pulmonary hypertension, atrial fibrillation, congestive heart failure and Eisenmenger's syndrome. The changes in the systemic vascular resistance (SVR) have important implication in the perioperative period in patients with ASD. We report the successful management of a 50year old female known to have osteum secundum type of ASD since childhood and who underwent Total Abdominal Hysterectomy under general anesthesia with transversus abdominis plane (TAP) block for postoperative analgesia. Perioperative anaesthesia management strategies are discussed.

\section{Case report}

A 50year old lady who is a known hypertensive with osteum secundum ASD with dysfunctional uterine bleeding for last 6 months was planned for Total Abdominal Hysterectomy. She also had a history of repeated respiratory tract infection and exertional dyspnea NHYA grade II. She was on Tab. Telmisartan 40mg once a day and Tab. Furosemide $40 \mathrm{mg}$ plus Tab. Amiloride $5 \mathrm{mg}$ once a day. On physical examination pulse was $78 / \mathrm{min}$ regular, good volume, noninvasive blood pressure was $130 / 80 \mathrm{mmHg}$ and normal jugular venous pressure. CVS examination revealed loud $\mathrm{P}$, ejection systolic murmur gr. IV/VI in pulmonary area. 12 lead ECG showed incomplete Right bundle branch block with right axis deviation. 2D Echo showed grossly dilated left and right atrium and right ventricle, large osteum secundum ASD (30 mm in diameter), left to right shunt, mild Tricuspid regurgitation, moderate pulmonary hypertension with an estimated right ventricle systolic pressure of $40 \mathrm{mmHg}$ and ejection fraction of $70.11 \%$. All blood investigations were within normal limits.

\section{Anaesthesia management}

After detailed pre-anaesthesia checkup, written informed consent was taken. Patient was kept nil orally for 6hours. Morning dose of oral Telmisartan was omitted. Injection: Ceftriaxone $2 \mathrm{gm}$ and Injection: Gentamicin $80 \mathrm{mg}$ intravenously was given preoperatively. Venous access was secured using an 18G IV cannula in left hand and ringer lactate was started. Routine monitoring of NIBP, Heart rate, ECG, $\mathrm{SpO}_{2}, \mathrm{ETCO}_{2} \mathrm{CVP}$ were done. Premedication with intravenous glycopyrrolate $0 . \mathrm{mg}$, midazolam $1 \mathrm{mg}$, dexamethasone $4 \mathrm{mg}$ and fentanyl 100ug was done. She was preoxygenated with $100 \%$ oxygen for 3 minutes. Induction was performed with Inj. Etomidate $15 \mathrm{mg}$ slow i.v and Inj. Vecuronium bromide $4 \mathrm{mg}$ i.v. Size-3 I-gel was inserted and patient was maintained on IPPV (Tidal volume- $400 \mathrm{ml}$, Respiratory rate- $12 / \mathrm{min}$ and PEEP $6 \mathrm{~cm} \mathrm{H}_{2} \mathrm{O}$ ) with $100 \%$ oxygen and sevoflurane to maintain MAC around 0.9 . The aim was to maintain end-tidal $\mathrm{CO}_{2}\left(\mathrm{ETCO}_{2}\right)$ around $32-35 \mathrm{mmHg}$. After induction Bilateral TAP block was performed by landmark approach using $22 \mathrm{G}$ blunt needle through the Triangle of Petit by loss of resistance (LOR) technique. $20 \mathrm{ml}$ of $0.2 \%$ Inj. Ropivacaine was injected in both sides.

Intraoperatively pulse varied between $60-80$ beats $/ \mathrm{min}$, blood pressure between $100 / 60-120 / 80 \mathrm{~mm} \mathrm{Hg}$ and $\mathrm{SpO}_{2}-100 \%$. During the $45 \mathrm{~min}$ procedure Lactated ringer's solution $500 \mathrm{ml}$ and normal saline was given. The urine output was $100 \mathrm{ml}$ and total blood loss was around $100 \mathrm{ml}$. Patient was reversed with neostigmine $2.5 \mathrm{mg}+$ glycopyrrolate $0.5 \mathrm{mg}$. After extubation patient was conscious, responding to verbal commands, with normal vitals and pain free. She was kept in recovery room for 1hour and then shifted to ICU. Postoperative analgesia was maintained by Intravenous Injection Paracetamol and Injection Diclofenac for one day then by oral analgesics. Patient was pain free 
postoperatively. Patient was orally allowed on second postoperative day on return of bowel function. Patient cardiac medication was resumed on the second postoperative day. She was discharged on $5^{\text {th }}$ postoperative day.

\section{Discussion}

Atrial septal defect (ASD) is an opening in the interatrial septum. It accounts for $6 \%-10 \%$ of all congential heart disease. ASD classification is based on ASD location relative to fossa ovalis, developmental anomaly and its size. Osteum secundum ASD accounts for $70 \%$ of cases which involves the fossa ovalis and is midseptal in location. ${ }^{2}$ ASD is the most common congential heart disease with a female to male ratio of 2:1. It is progressive in nature so the patients are usually asymptomatic during childhood. However, with increasing age due to reversal of shunt symptoms appear later in life and by age $40,90 \%$ of untreated patients have symptoms of exertional dyspnea, fatigue, palpitation or sustained arrhythmia. ${ }^{3}$ Complications of uncorrected secundum type of ASD include pulmonary arterial hypertension, right sided heart failure, atrial fibrillation/flutter, stroke and Eisenmenger's syndrome. ${ }^{4}$

Large ASD (>9mm), may result in a clinically remarkable left-toright shunt. The adverse effect of persistently increased pulmonary blood flow leads to increased in pulmonary vascular resistance and subsequent pulmonary arterial hypertension, cardiomegaly, arrthymias and myocardial ischemia. Pulmonary hypertension is classified as mild $(36-49 \mathrm{mmHg})$, moderate $(50-59 \mathrm{mmHg})$, severe $(60 \mathrm{mmHg})$ according with right ventricular systolic pressure calculated by echocardiography. ${ }^{5}$ The echocardiography helps to establish the size and location of the ASD, magnitude and hemodynamic impact of the left to right shunt, and the presence and the degree of pulmonary hypertension. ${ }^{6,7}$ Perioperative change in SVR can have important implications in patients with ASD. ${ }^{8}$ The magnitude of left to right shunt depends on size of ASD, ventricular diastolic properties and the relative impedance in pulmonary and systemic circulation. ${ }^{9}$ The problems which are anticipated during general anesthesia in these patients are air embolism during vascular access, heart block, dysrhythmias (5\%-10\%), heart failure and infective endocarditis.

Our patient is a 50year old lady, a known case of Osteum secundum ASD who underwent Total Abdominal hysterectomy. The anaesthesia techniques which are have been commonly used for abdominal hysterectomy in these types of patients is usually general anaesthesia with epidural anesthesia. However, these techniques carry the risk of sudden and uncontrolled hypotension and unstable hemodynamics with the possibility of reversal of intra cardiac shunt. So we employed a unique anaesthesia approach of General Anaesthesia with controlled ventilation and bilateral TAP block with particular attention to control of factors that may cause sudden pulmonary hypertension and resultant hypoxemia and the potential for the development of acute heart failure, which would have been detrimental to the patient. Our patient had moderate pulmonary hypertension $100 \%$ oxygen was beneficial for her. The use of fentanyl and sevoflurane with the benefits of amnesia and analgesia permitted us to use 100\% oxygen. We used Injection Etomidate as induction agent for better haemodynamic stability. I-gel was used to avoid sympathetic stimulation during laryngoscopy and intubation which would have caused increased SVR, hypertension and detrimental effect on the left to right shunt. Hypercarbia can be avoided by adequate adjustment of tidal volume and respiratory rate. The use of mechanical ventilation causes stretching of lungs leading to release of endogenous nitric oxide and prostaglandins which cause pulmonary vasodilatation. ${ }^{10}$ Intraoperatively we avoided factors that can increase the shunt, maintained adequate preload and cardiac contractility, a near normal heart rate, SVR and PVR. Adequate monitoring was done to detect atrial arrhythmias and to avoid hypercarbia and hypoxia.

The Bilateral TAP block with NSAIDs provided superior postoperative analgesia without causing any haemodynamic instability. ${ }^{11}$ The use of epidural catheter for postoperative analgesia might have caused unstable haemodynamics which could have been detrimental to our patient. ${ }^{12,13}$ Postoperatively our aim was to avoid hypoxia and adequate pain control which was achieved by giving postoperative oxygen by mask for 4 hours and bilateral TAP block. This combined technique of General anaesthesia with bilateral TAP block can be used in these type of patients as its provides better intraoperative stability and good postoperative analgesia without causing haemodynamic instability.

\section{Conclusion}

Patients with ASD can undergo non cardiac surgery with the use of adequate balanced anaesthesia plan which can avoid increase in pulmonary vascular resistance, decrease in systemic vascular resistance, tachycardia, hypotension, hypercarbia, hypoxia and hypothermia. We achieved this balanced anesthesia by using general anaesthesia and bilateral TAP block. To conclude, with good preoperative assessment, proper preparation and providing good intraoperative and postoperative analgesia non-cardiac surgeries can be easily performed in these type of patients.

\section{Acknowledgements}

None.

\section{Conflicts of interest}

The authors declare that there are no conflicts of interest.

\section{Funding}

None.

\section{References}

1. Feldt RH, Avasthey P, Yoshimasu F, et al. Incidence of congenital heart disease in children born to residents of Olmsted County, Minnesota, 1950-1969. Mayo Clin Proc. 1971;46(12):794-799.

2. Brickner ME, Hillis LD, Lange RA. Congenital heart disease in adults. N Engl J Med. 2000;342(4):256-263.

3. Saxena A, Divekar A, Soni NR. Natural history of secundum atrial septal defect revisited in the era of transcatheter closure. Indian Heart. 2005;57(1):35-38.

4. Balint $\mathrm{OH}$, Samman A, Haberer K, et al. Outcomes in patients with pulmonary hypertension undergoing percutaneous ASD closure. BMJ Heart. 2008;94(9):1189-1193.

5. Valdes Cruz LM, Cayre RO. Anomalies of right ventricular outflow tract and pulmonary arteries. In: Valdes-Cruz LM \& Cayre RO, Editors. Philadelphia, USA: Lippincott-Raven; 1999. pp.325-348.

6. Lee MG, Ko JS, Yoon HJ, et al. An unusual presentation of an atrial septal defect. J Cardiovasc Ultrasound. 2009;17(4):151-152.

7. Hines RL, Marschall KE. Stoelting's anesthesia and co-existing disease. 6th edn. In: Livingstone C, Editor. India: Elsevier; 2008. p. 60.

8. Siu SC, Sermer M, Colman JM, et al. Prospective multicentre study of pregnancy outcomes in womens with heart disease. Circulation. 2001;104(5):515-521. 
9. Morray JP, Lynn AM, Mansfield PB. Effect of $\mathrm{pH}$ and $\mathrm{PaCO}_{2}$ on pulmonary and systemic haemodynamics after surgery in children with congenital heart disease and pulmonary hypertension. $J$ Paediatr. 1998;113(3):474-479.

10. Mark J Young, Andrew W Gorlin, Vicki E Modest, et al. Clinical Implications of Transverse Abdominis Plane Block in Adults. Anesthesiology Research and Practice. 2012;731645.

11. Wahba SS, Kamal SM. Analgesic efficacy and outcome of transversesabdominis plane block versus low thoracic-epidural analgesia after laprotomy in ischemic heart disease patients. J Anesth. 2014;28(4):517523.
12. Champaneria R, Shah L, Geoghegan J, et al. Analgesic effectiveness of transversus abdominis plane block after hysterectomy:a meta-analysis. EJOGRB. 2013;166(1):1-9.

13. Weiss BM, Hess OM. Pulmonary vascular disease and pregnancy:current controversies, management strategies, and perspectives. Eur Heart J. 2000;21(2):104-115. 